\title{
Transanal Endoscopic Microsurgical Submucosal Dissection: an Efficient Treatment Option for Giant Superficial Neoplastic Lesions of The Rectum
}

Konstantinos Kouladouros ( $\sim$ kouladou@otenet.gr)

University Medical Centre Mannheim

Jörg Baral

Städtisches Klinikum Karlsruhe

\section{Research Article}

Keywords: Rectal adenoma, Early rectal cancer, Transanal surgery, Endoscopic submucosal dissection, Transanal endoscopic microsurgery, Minimally invasive surgery

Posted Date: August 17th, 2021

DOI: https://doi.org/10.21203/rs.3.rs-799168/v1

License: (c) (i) This work is licensed under a Creative Commons Attribution 4.0 International License.

Read Full License 


\section{Abstract}

\section{Purpose}

The resection of giant superficial neoplastic lesions of the rectum $(>5 \mathrm{~cm})$ is challenging and the ideal resection technique remains a controversial issue. Aim of our study is to assess the feasibility and outcomes of transanal endoscopic microsurgical submucosal dissection (TEM-ESD), a new hybrid technique, for the resection of giant superficial rectal neoplasms.

\section{Methods}

We retrospectively analyzed all cases of TEM-ESD for superficial rectal tumors $>5 \mathrm{~cm}$ performed in the Department of Surgery of the Municipal Hospital of Karlsruhe between 2010 and 2020.

\section{Results}

We identified 43 cases matching our criteria (35 adenomas, 8 adenocarcinomas). The median size of the lesions was $75 \mathrm{~mm}$, the median operating time was $81.5 \mathrm{~min}$. En bloc resection was possible in all cases. In a follow-up period of 15 months there were 2 cases of local recurrence.

\section{Conclusions}

TEM-ESD is a feasible and safe therapeutic option for the treatment of giant superficial rectal neoplasms.

\section{Introduction}

Superficial neoplastic lesions of the rectum, including adenomas and early adenocarcinomas, are among the most common colorectal neoplasms ${ }^{1}$. Conventional endoscopic polypectomy and endoscopic mucosal resection (EMR) are adequate therapeutic options with en bloc resection and low local recurrence rates for lesions up to $3 \mathrm{~cm}^{2}$. However, the optimal treatment of tumors larger than $5 \mathrm{~cm}$ is still unclear $^{3-5}$. Piecemeal EMR is a common, quick and safe therapeutic option in such cases, but it has been associated with insufficient histological assessment of the specimen and increased risk of local recurrence, ranging from 14 to $25 \%{ }^{6-8}$.

Transanal endoscopic microsurgery (TEM) was introduced in the 1980s and enabled surgeons to perform en bloc resections of larger lesions in the entire rectum ${ }^{9}$. Full-thickness resection, which is the treatment of choice for most specialists, allows for optimal resection margins for potentially malignant lesions, but at the same time compromises the mesorectum and is associated with an increased risk of local complications ${ }^{10,11}$. More recently, endoscopic submucosal dissection (ESD), initially described in Japan for the treatment of early gastric cancer, was proposed as an alternative method for the en bloc resection of large colorectal adenomas ${ }^{12}$. Colorectal ESD is, nevertheless, a time consuming and technically 
demanding procedure, associated with an increased risk of perforation, especially when addressing larger lesions, and therefore it has not gained wide acceptance in the Western world yet ${ }^{13-16}$.

Transanal endoscopic microsurgical submucosal dissection (TEM-ESD) is a new resection technique that combines the technical advantages of the rigid TEM instruments with the minimally invasive approach of ESD. This technique has been successfully used for the treatment of rectal adenomas and low-risk carcinomas and has been associated with low morbidity and local recurrence rates ${ }^{17}$. The application of traction through an independently controlled grasper while performing the submucosal dissection is the key feature of this technique and its advantages become even more obvious during the resection of challenging lesions, such as giant tumors and tumors in difficult localizations. However, the outcomes of TEM-ESD in these selected patient groups have not been studied yet.

The aim of our study is to analyze the feasibility and outcomes of TEM-ESD for the resection of giant rectal lesions with a maximum diameter of more than $50 \mathrm{~mm}$.

\section{Materials And Methods}

We retrospectively analyzed all patients undergoing TEM-ESD in the Department of Surgery of the Municipal Hospital of Karlsruhe between 2010 and 2020 and we included all cases of rectal adenomas with a diameter of more than $5 \mathrm{~cm}$ according to the postoperative pathology report.

The preoperative staging included flexible endoscopy and transrectal ultrasound or MRI to rule out an invasion beyond the submucosal layer. All the surgical procedures were performed after complete bowel preparation and under general anesthesia, using the original technique as in its initial description ${ }^{17}$.

In cases of occult carcinomas, the pathological findings were discussed in the multidisciplinary gastrointestinal tumor board. Low-risk early rectal cancer was defined as pT1 sm1-2, G1-2, L0, V0. Complete en bloc resections (R0) of low-risk tumors were considered curative and no further treatment was necessary, whereas high-risk tumors required further oncologic treatment with radical surgery and total mesorectal excision (TME).

Demographical, diagnostic, perioperative, histological and follow-up data were retrospectively entered in an electronic database after pseudonymization. All methods were performed in accordance with the relevant guidelines and regulations. Approval from the Ethics Committee of the University of Heidelberg was acquired.

The following were analyzed: demographic characteristics; endoscopic findings including size, localization and morphology of the lesion; total operative time; postoperative adverse events including their treatment; histopathological findings including resection status; overall hospital stay; and follow-up data. 
The statistical analysis was performed with SigmaStat 3.0 (Systat Software GmbH). For variables approximately normally distributed (i.e. age), mean and standard deviation were calculated. Skewed data are presented by their median together with the range. For categorical factors, absolute and relative frequencies are given together with exact $95 \%$ confidence intervals $(\mathrm{Cl})$. The statistical review of the study was performed by a biomedical statistician.

\section{Results}

Out of a total of 192 procedures, we were able to identify 43 cases fulfilling our inclusion criteria. Twentyseven patients were male and 16 were female. The mean age was $70.4( \pm 10.46)$ years, ranging from 50 to 90 years. Twelve lesions were detected in screening colonoscopy, eight in follow-up colonoscopy, 14 presented with rectal bleeding and nine with alteration of the bowel function. Three of the lesions were recurrent adenomas after endoscopic mucosal resection (EMR).

The median size of the lesions, as described in the postoperative pathology report, was $75 \mathrm{~mm}$ (range: $51-185 \mathrm{~mm}$ ). The median distance of the anal margin of the lesion to the anal verge was $6 \mathrm{~cm}$ (range: $0-15 \mathrm{~cm})$. The exact distribution of the lesions in the rectum is depicted in Table 1.

Table 1

Localization of the lesions

\begin{tabular}{|ll|}
\hline Localization & No of cases (\%) \\
\hline Upper rectum & $2(4.7 \%)$ \\
\hline Mid rectum & $20(46.5 \%)$ \\
\hline Lower rectum & $21(48.8 \%)$ \\
\hline Involvement of the dentate line & $6(14.0 \%)$ \\
\hline Anterior wall & $10(23.3 \%)$ \\
\hline Posterior wall & $15(34.9 \%)$ \\
\hline Right lateral wall & $10(23.3 \%)$ \\
\hline Left lateral wall & $8(18.6 \%)$ \\
\hline
\end{tabular}

The median operating time was 81.5 min (range: $30-340 \mathrm{~min}$ ). Five patients (11.6\%; Cl: [4\%; 25\%]) presented with delayed bleeding, two of which could be treated conservatively, two required an endoscopic intervention and one required a transanal surgical revision. No other adverse events were recorded. Thus, the major complication rate (>IIla according to the Dindo-Clavien classification) was $2.3 \%$ $(\mathrm{Cl}:[0 ; 12])^{18}$. The median overall hospital stay was four days (range: $2-8$ days).

The histological features of the resected lesions are summarized in Table 2. The histopathological report revealed an occult adenocarcinoma in eight (18.6\%) cases. According to their histological features, four 
were low risk and the remaining four were high risk.

Table 2

Histological features

\begin{tabular}{|ll|}
\hline Histological Feature & No of cases (\%) \\
\hline Adenoma & $35(81.4 \%)$ \\
\hline Tubular & $4(9.3 \%)$ \\
\hline Villous & $4(9.3 \%)$ \\
\hline Tubulovillous & $27(62.8 \%)$ \\
\hline Traditional serrated & $1(2.3 \%)$ \\
\hline Low grade dysplasia & $11(25.6 \%)$ \\
\hline High grade dysplasia & $24(55.8 \%)$ \\
\hline Adenocarcinoma & $8(18.6 \%)$ \\
\hline pT1sm1 & $4(9.3 \%)$ \\
\hline pT1sm3 & $4(9.3 \%)$ \\
\hline L0 and V0 & $7(16.3 \%)$ \\
\hline L1 and V1 & $1(2.3 \%)$ \\
\hline G1 & $2(4.7 \%)$ \\
\hline G2 & $6(14.0 \%)$ \\
\hline
\end{tabular}

All resections were completed using the TEM-ESD technique and no conversions to other methods were necessary. All lesions were resected en bloc and macroscopically completely. The postoperative pathology report confirmed a complete en bloc resection in 29 (67.4\%, Cl: [51\%; 81\%]) cases. All adenomas had a free deep resection margin, however in 13 cases (37.1\% of the adenomas, Cl: [21\%; $55 \%]$ ) the tears on the lateral margin did not allow for an accurate evaluation of the resection status. Seven out of eight carcinomas were resected completely (R0). The lateral margin was free in all eight cases, whereas the deep margin was positive in one case of deep submucosa infiltrating carcinoma (sm3). The resection was curative in all cases of low-risk carcinomas and in $50 \%$ of all carcinomas. The interdisciplinary tumor board set the indication for oncologic surgical resection in all cases of high-risk carcinomas, however all four patients refused to undergo any further treatment.

The median follow-up was 15 months. Three patients (7.0\%; Cl: [1.5\%; 19\%]) developed a stenosis, which was successfully treated with endoscopic balloon dilation. All three patients had lesions involving more than $3 / 4$ of the rectal wall circumference. No recurrence was recorded among the patients with adenomas. One patient with a curative resection of a low-risk carcinoma presented with a local recurrence six months 
after surgery and could be treated successfully with a full-thickness TEM. Among the high-risk carcinomas, one local recurrence was reported, but the patient refused any further treatment.

\section{Discussion}

The local resection of giant rectal adenomas and early adenocarcinomas is a controversial issue and the ideal technique for that has yet to be determined. Most authors define giant rectal lesions as those with a maximum diameter of more than $5 \mathrm{~cm}^{4,5}$. Very few studies have addressed the en bloc resection of these lesions, mostly using TEM and more recently also ESD. These studies have shown the feasibility of those methods, but at the same time they pointed out the high risk of tissue fragmentation and adverse events or the high level of technical difficulty of the procedures ${ }^{19-23}$. That is probably the reason why many of these lesions are still referred for radical surgical treatment, including anterior resection with total mesorectal excision (TME) or abdominoperineal resection (APR) ${ }^{24}$. The findings of our study suggest that TEM-ESD is a feasible therapeutic alternative for the local resection of giant rectal lesions, offering a high en bloc resection rate with minimal morbidity and optimal long-term results.

The minimally invasive approach and low recurrence rates of ESD make this technique very promising, however the lack of traction is considered to be one of its main technical disadvantages. Various traction methods and devices have been described in order to allow easier access to the submucosal plane, none of which has gained wide acceptance to date ${ }^{25-27}$. In the case of large lesions, visualization can be obstructed by the flap, which significantly increases the total procedure time and sometimes does not allow for completion of the procedure ${ }^{13}$. TEM-ESD addresses this issue by introducing traction with a second, independently controlled, rigid instrument during submucosal dissection. This innovation facilitates both visualization and dissection and thus reduces overall procedure time. In our study, median operating time was $81.5 \mathrm{~min}$ and thus $35-50 \%$ shorter than those reported for ESD 20,21 .

In terms of morbidity, in our study TEM-ESD showed an acceptable safety profile, with overall morbidity of $8 \%$ and major complications occurring in only one case. Our findings are similar to previous reports for smaller lesions, implying that size might not increase the periprocedural risk ${ }^{17}$. We consider this factor to be a major advantage of TEM-ESD, since flexible ESD has been associated with a significantly higher risk of adverse events when used for the treatment of lesions $>5 \mathrm{~cm}$, reaching morbidity rates of up to $44 \%$ for lesions $>10 \mathrm{~cm}^{13,19}$. The same principle applies to TEM, with morbidity rates of $26.5 \%$ for lesions $5-8$ $\mathrm{cm}$ and up to $36.1 \%$ for lesions larger than $8 \mathrm{~cm}^{22}$. In particular, dehiscence of the suture line, reported in $15 \%$ of full-thickness TEM resections for large tumors, is not an issue when performing TEM-ESD, since the M. propria layer remains intact and no closure of the defect is necessary ${ }^{28}$.

Another important feature of TEM-ESD is its ability to achieve high en bloc resection rates. This feature seems to apply for large lesions as well, since an en bloc resection was possible in all cases in our study. The importance of en bloc resection of rectal adenomas and its direct association with low recurrence rates has often been pointed out in the literature ${ }^{7}$. Additionally, the risk of occult invasive cancer is 
significantly higher in large rectal adenomas, rising up to $20 \%$ for lesions $>50 \mathrm{~mm}$ according to the literature ${ }^{21,22,29}$. Our findings confirm those results, as we were able to detect an adenocarcinoma in $18.6 \%$ of our cases. En bloc resection of these potentially malignant lesions allows for an accurate estimation of invasion depth and evaluation of the deep resection margin, and thus for adequate staging ${ }^{21,30}$. In the case of low-risk tumors, a complete en bloc resection is curative and spares the patient a major surgical procedure. TEM was initially introduced as a technique that enables en bloc resection of rectal lesions, however the risk of fragmentation seems to increase significantly for larger tumors and en bloc resection is possible in $63-87 \%$ of cases according to different studies ${ }^{22,23}$. ESD has been reported to achieve higher en bloc resection rates, comparable to those reported in our study ${ }^{21}$.

Our data show a discrepancy between en bloc and complete en bloc resections, with the latter being histologically confirmed in $67.1 \%$ of the specimens. Although this difference is not as obvious after conventional TEM, it has been reported frequently after ESD, but has not been associated with higher recurrence rates $22,31,32$. These findings are probably a result of the fragility of the ESD specimens, which do not include the M. propria and are thus susceptible to tears. These artifacts do not allow for an exact evaluation of the lateral margins and decrease the complete en bloc resection rates. In the case of TEM$E S D$, the manipulation of the lateral specimen margins with the grasper during submucosal dissection is an additional risk factor leading to such artifacts, especially influencing large lesions, requiring frequent repositioning of the instruments to achieve optimal traction. Nevertheless, a complete resection still seems to be possible in most cases, even if not histologically confirmed, as suggested by the low local recurrence rates.

No recurrence was reported after resection of adenomas in our study. There was only one case of local recurrence after curative resection of a low-risk adenocarcinoma, which could be successfully treated with a full-thickness TEM procedure. Interestingly enough, there was only one case of local recurrence among the patients with high-risk adenocarcinomas refusing further treatment, although the small number of cases does not allow for solid conclusions about the prognosis of such lesions. Similar longterm results have been reported after ESD for large rectal lesions ${ }^{13,20}$. On the contrary, recurrence rates range from $11-25 \%$ after TEM, probably because of the higher risk of fragmentation during the resection, implying a long-term advantage of submucosal dissection techniques ${ }^{23,33}$.

Postoperative strictures have been reported as long-term adverse events after resection of large tumors in different parts of the gastrointestinal tract, especially if they involve more than $50 \%$ of the circumference. In the case of ESD, this is a major issue after resection of esophageal lesions, but symptomatic strictures are less common in the colorectum, probably because of the size of the lumen ${ }^{21}$. The risk of stenosis increases with the size of the lesion, but the most important factor seems to be the involvement of more than $75 \%$ of the circumference, in which case the rate of stenosis is almost $20 \% 34$. The same applies for TEM, after which the risk of stenosis is estimated to be $7 \%$ after resection of lesions between 5 and $8 \mathrm{~cm}$ and can reach $23 \%$ for circumferential lesions ${ }^{22,35}$. In our study we had three cases of stricture, all after 
resection of lesions involving more than $3 / 4$ of the circumference. All cases could be treated endoscopically, confirming the findings of other authors ${ }^{34}$.

Our study is the first to analyze the periprocedural and long-term outcomes of TEM-ESD for the resection of giant rectal adenomas and early adenocarcinomas. The number of patients included is larger than that of most similar studies published to date and the report of all consecutive cases in a 10-year period reduces selection bias ${ }^{13,20,23,33}$. Nevertheless, the retrospective and observational design, as well as the rather small sample size of the study, remains a significant limitation. The small number of such resections performed even in specialized centers makes a prospective comparison of different techniques difficult to perform, especially since very few centers have the necessary expertise in more than one of these techniques. With increasing experience, a multi-center, prospective, randomized study will be able to give a definitive answer to the question of the ideal resection technique in the future.

\section{Conclusions}

TEM-ESD appears to be a safe and feasible treatment option for giant rectal adenomas and early adenocarcinomas. Traction and triangulation allow for a quick and accurate resection of these challenging lesions, achieving high rates of en bloc resections. The large size of the lesions does not seem to increase periprocedural morbidity, and long-term adverse events, such as strictures, are rare and can be treated endoscopically. Most importantly, TEM-ESD offers excellent long-term outcomes with low recurrence rates for both adenomas and low-risk adenocarcinomas. Taking all these factors into consideration, we believe that TEM-ESD is the optimal treatment for large rectal lesions, particularly demonstrating its technical advantages in this challenging group of patients.

\section{Declarations}

Conflicts of Interest: Jörg Baral declares he has a consulting contract with Richard Wolf GmbH, Knittlingen, Germany. Konstantinos Kouladouros has none to declare.

Funding: This research did not receive any specific grant from funding agencies in the public, commercial, or not-for-profit sectors.

Availability of data and material: Upon special request and after approval of the local ethics committee

Code Availability: Not applicable

Author's contributions: Both authors contributed equally to the conception of the study and the data analysis. J. Baral first described the technique studied and performed most of the resections. K. Kouladouros wrote the manuscript. Both authors revised and approved the manuscript.

Ethics Statement / Consent to participate / Consent for publication: Approval from the local Ethics Committee (University of Heidelberg) was acquired. All data were pseudonymized and retrospectively 
analyzed, so that no informed consent was necessary according to the Ethics Committee.

\section{References}

1. Jahadi, M. R. \& Baldwin, A. Villous adenomas of the colon and rectum. The American Journal of Surgery, 130, 729-732 (1975).

2. Denzer, U. et al. S2k-Leitlinie Qualitätsanforderungen in der gastrointestinalen Endoskopie, AWMF Register Nr. 021-022. Zeitschrift für Gastroenterologie, 53, E1-E227 (2016).

3. Antillon, M. R. et al. En bloc endoscopic submucosal dissection of a 14-cm laterally spreading adenoma of the rectum with involvement to the anal canal: expanding the frontiers of endoscopic surgery (with video). Gastrointest. Endosc, 67, 332-337 (2008).

4. Dulskas, A., Kilius, A., Petrulis, K. \& Samalavicius, N. E. Transanal endoscopic microsurgery for giant benign rectal tumours: is large size a contraindication? International Journal of Colorectal Disease, 32, 1759-1761 (2017).

5. Scala, A., Gravante, G., Dastur, N., Sorge, R. \& Simson, J. N. L. Transanal endoscopic microsurgery in small, large, and giant rectal adenomas. Archives of Surgery, 147, 1093-1100 (2012).

6. Lee, E. J., Lee, J. B., Lee, S. H. \& Youk, E. G. Endoscopic treatment of large colorectal tumors: Comparison of endoscopic mucosal resection, endoscopic mucosal resection-precutting, and endoscopic submucosal dissection. Surg. Endosc, 26, 2220-2230 (2012).

7. Saito, Y. et al. Clinical outcome of endoscopic submucosal dissection versus endoscopic mucosal resection of large colorectal tumors as determined by curative resection. Surg. Endosc, 24, 343-352 (2010).

8. Kobayashi, N. et al. Matched case-control study comparing endoscopic submucosal dissection and endoscopic mucosal resection for colorectal tumors. Journal of Gastroenterology and Hepatology (Australia), 27, 728-733 (2012).

9. Buess, G., Theiss, R., Gunther, M., Hutterer, F. \& Pichlmaier, H. Endoscopic surgery in the rectum. Endoscopy, 17, 31-35 (1985).

10. Allaix, M. E. et al. Transanal Endoscopic Microsurgery for Rectal Neoplasms. How I Do It. Journal of Gastrointestinal Surgery, 17, 586-592 (2013).

11. Kumar, A. S. et al. Complications of transanal endoscopic microsurgery are rare and minor: A single institution's analysis and comparison to existing data. Diseases of the Colon and Rectum, 56, 295300 (2013).

12. Yamamoto, H. et al. Successful en-bloc resection of large superficial tumors in the stomach and colon using sodium hyaluronate and small-caliber-tip transparent hood. Endoscopy, 35, 690-694 (2003).

13. Jung, D. H., Youn, Y. H., Kim, J. H. \& Park, H. Endoscopic submucosal dissection for colorectal lateral spreading tumors larger than $10 \mathrm{~cm}$ : Is it feasible? Gastrointest. Endosc, 81, 614-620 (2015). 
14. Tanaka, S. et al. Endoscopic submucosal dissection for colorectal neoplasia: possibility of standardization. Gastrointest. Endosc, 66, 100-107 (2007).

15. Probst, A. et al. Endoscopic submucosal dissection for early rectal neoplasia: Experience from a European center. Endoscopy, 49, 222-232 (2017).

16. Wagner, A. et al. Single-center implementation of endoscopic submucosal dissection (ESD) in the colorectum: Low recurrence rate after intention-to-treat ESD. Digestive Endoscopy, 30, 354-363 (2018).

17. Baral, J. Transanal endoscopic microsurgical submucosa dissection in the treatment of rectal adenomas and T1 rectal cancer. Coloproctology vol. 40 364-372(2018).

18. Dindo, D., Demartines, N. \& Clavien, P. A. Classification of Surgical Complications. Annals of surgery, 240, 205-213 (2004).

19. Saito, Y. et al. A prospective, multicenter study of 1111 colorectal endoscopic submucosal dissections (with video). Gastrointest. Endosc, 72, 1217-1225 (2010).

20. Tang, X. W. et al. Endoscopic submucosal dissection for laterally spreading tumors in the rectum $\geq$ $40 \mathrm{~mm}$. Techniques in Coloproctology, 20, 437-443 (2016).

21. Hayashi, Y. et al. Efficacy and safety of endoscopic submucosal dissection for superficial colorectal tumors more than $50 \mathrm{~mm}$ in diameter. Gastrointest. Endosc, 83, 602-607 (2016).

22. Serra-Aracil, X. et al. Transanal endoscopic microsurgery in very large and ultra large rectal neoplasia. Techniques in Coloproctology, 23, 869-876 (2019).

23. Levic, K., Bulut, O. \& Hesselfeldt, P. Transanal endoscopic microsurgery for giant polyps of the rectum. Techniques in Coloproctology, 18, 521-527 (2014).

24. Mounzer, R. et al. Endoscopic and surgical treatment of malignant colorectal polyps: A populationbased comparative study. Gastrointest. Endosc, 81, 733-7402 (2015).

25. Uraoka, T. et al. Thin endoscope-assisted endoscopic submucosal dissection for large colorectal tumors (with videos) $\{$ A figure is presented\}. Gastrointest. Endosc, 66, 836-839 (2007).

26. Motohashi, O. Two-point fixed endoscopic submucosal dissection in rectal tumor (with video). Gastrointest. Endosc, 74, 1132-1136 (2011).

27. Yamasaki, Y. et al. Efficacy of traction-assisted colorectal endoscopic submucosal dissection using a clip-and-thread technique: A prospective randomized study. Digestive Endoscopy, 30, 467-476 (2018).

28. Schäfer, H., Baldus, S. E. \& Hölscher, A. H. Giant adenomas of the rectum: Complete resection by transanal endoscopic microsurgery (TEM). International Journal of Colorectal Disease, 21, 533-537 (2006).

29. Kaku, E. et al. Proportion of Flat- and Depressed-Type and Laterally Spreading Tumor Among Advanced Colorectal Neoplasia. Clinical Gastroenterology and Hepatology, 9, 503-508 (2011).

30. Saito, Y. et al. Endoscopic treatment of large superficial colorectal tumors: a case series of 200 endoscopic submucosal dissections (with video)\{A figure is presented\}. Gastrointest. Endosc, 66, 
966-973 (2007).

31. Niimi, K. et al. Long-term outcomes of endoscopic submucosal dissection for colorectal epithelial neoplasms. Endoscopy, 42, 723-729 (2010).

32. Isomoto, H. et al. Clinicopathological factors associated with clinical outcomes of endoscopic submucosal dissection for colorectal epithelial neoplasms. Endoscopy, 41, 679-683 (2009).

33. Khoury, R., Duek, S. D., Issa, N. \& Khoury, W. Transanal endoscopic microsurgery for large benign rectal tumors; where are the limits? International Journal of Surgery, 29, 128-131 (2016).

34. Ohara, Y. et al. Risk of stricture after endoscopic submucosal dissection for large rectal neoplasms. Endoscopy, 48, 62-70 (2016).

35. Arezzo, A. et al. Transanal endoscopic microsurgery for giant circumferential rectal adenomas. Colorectal Dis, 18, 897-902 (2016). 